\title{
Síntese verde e caracterização de nanopartículas de prata em curcumina e extrato de cajueiro (Anacardium occidentale)
}

\author{
Green synthesis and characterization of silver nanoparticles in curcumin and cashew extract \\ (Anacardium occidentale)
}

Síntesis verde y caracterización de nanopartículas de plata en curcumina y extracto de anacardo (Anacardium occidentale)

Recebido: 26/04/2021 | Revisado: 05/05/2021 | Aceito: 07/05/2021 | Publicado: 22/05/2021

\author{
Alciomar Veras Viana \\ ORCID: https://orcid.org/0000-0002-4928-5452 \\ Centro Universitário UniFacid, Brasil \\ E-mail: alciomar@naeon.org.br \\ Deuzuíta dos Santos Freitas Viana \\ ORCID: https://orcid.org/0000-0002-1902-6505 \\ Centro Universitário Unifacid, Brasil \\ E-mail: deuzuitasfv@gmail.com \\ Girlene Soares de Figueirêdo \\ ORCID: https://orcid.org/0000-0002-6710-1549 \\ Universidade Federal do Piauí, Brasil \\ E-Mail: girlenesf@gmail.com \\ Josimar Elpídio de Brito \\ ORCID: https://orcid.org/0000-0001-7323-5511 \\ Centro Universitário UniFacid, Brasil \\ E-Mail: josimarelpidio8@gmail.com \\ Vicente Galber Freitas Viana \\ ORCID: https://orcid.org/0000-0002-3863-6974 \\ Instituto Federal de Educação, Ciência e Tecnologia do Piauí, Brasil \\ E-Mail: galber@ifpi.edu.br
}

\begin{abstract}
Resumo
Esta pesquisa teve como objetivo sintetizar, caracterizar e comparar a formação de nanopartículas de prata em Curcuma longa e Anacardium occidentale. As soluções hidroalcoólicas foram preparadas com $200 \mathrm{~g}$ do pó de curcumina e folhas de cajueiro respectivamente separadas, misturada com álcool a 70\%, para obtenção das nanopartículas utilizou nitrato de prata $1,0 \mathrm{mmol} / \mathrm{L}$, citrato de sódio $1 \%$ e adicionou o extrato de curcumina e o extrato de folhas de cajueiro, separadamente. As nanopartículas foram caracterização por espectroscopia infravermelha em um feixe de comprimento de onda entre 250 a $800 \mathrm{~nm}$. Verificou-se por meio do pico de absorção atribuído a Ressonância Plasmônica de Superfície (SPR) que tanto o extrato de curcumina, quanto no extrato de cajueiro foram capazes de sintetizar e estabilizar as nanopartículas de prata com formas esféricas e com tamanho aproximadas de 20nm. Os dois extratos vegetais utilizados se mostraram eficientes no desenvolvimento e estabilização de nanopartículas de prata por meio da síntese verde.
\end{abstract}

Palavras-chave: Nanoestruturas; Química verde; Composto de prata.

\begin{abstract}
This research aimed to synthesize, characterize and compare the formation of silver nanoparticles in Curcuma longa and Anacardium occidentale. The hydroalcoholic solutions were prepared with $200 \mathrm{~g}$ of curcumin powder and cashew leaves respectively, mixed with $70 \%$ alcohol, to obtain the nanoparticles, it used $1.0 \mathrm{mmol} / \mathrm{L}$ silver nitrate, $1 \%$ sodium citrate and added the curcumin extract. and the cashew leaf extract, separately. The nanoparticles were characterized by infrared spectroscopy in a beam with a wavelength between 250 and $800 \mathrm{~nm}$. It was verified by means of the absorption peak attributed to Plasmonic Surface Resonance (SPR) that both the curcumin extract and the cashew extract were able to synthesize and stabilize the silver nanoparticles with spherical shapes and approximately $20 \mathrm{~nm}$ in size. The two plant extracts used were shown to be efficient in the development and stabilization of silver nanoparticles by means of green synthesis.
\end{abstract}

Keywords: Nanostructures; Green chemistry; Silver compound. 


\begin{abstract}
Resumen
Esta investigación tuvo como objetivo sintetizar, caracterizar y comparar la formación de nanopartículas de plata en Curcuma longa y Anacardium occidentale. Las soluciones hidroalcohólicas se prepararon con $200 \mathrm{~g}$ de curcumina en polvo y hojas de anacardo respectivamente, mezcladas con alcohol al 70\%, para obtener las nanopartículas, se utilizó nitrato de plata $1.0 \mathrm{mmol} / \mathrm{L}$, citrato de sodio al $1 \%$ y se agregó el extracto de curcumina. Y el extracto de hoja de anacardo, por separado. Las nanopartículas se caracterizaron mediante espectroscopía infrarroja en un haz con una longitud de onda entre 250 y $800 \mathrm{~nm}$. Se verificó mediante el pico de absorción atribuido a la Resonancia de Superficie Plasmónica (SPR) que tanto el extracto de curcumina como el extracto de anacardo eran capaces de sintetizar y estabilizar las nanopartículas de plata con formas esféricas y de aproximadamente $20 \mathrm{~nm}$ de tamaño. Los dos extractos de plantas utilizados demostraron ser eficientes en el desarrollo y estabilización de nanopartículas de plata mediante síntesis verde.
\end{abstract}

Palabras clave: Nanoestructuras; Química verde; Compuesto de plata.

\title{
1. Introdução
}

Os recentes avanços da nanotecnologia permitiu um maior controle da matéria devido à possibilidade de se produzir materiais em escalas manométricas de dimensões entre 1 a 100nm (Coura et al., 2018). Com isso, a nanotecnologia ganhou notoriedade e impactou vários aspectos no campo industrial e tecnológico. Diferentemente do seu estado natural e, dependendo das características geométricas, área superficial, forma e composição química as nanopartículas se comportam de formas diferentes (Echeverry-Chica, 2020).

Os nanomateriais despertam o interesse de varias áreas das ciências, entre eles as nanopartículas de prata (AgNPs), se destacam pela alta estabilidade térmica, versatilidade, ação de longo prazo, durabilidade e pelo seu desempenho antimicrobiano. Além disso, pode ser sintetizadas em diferentes substratos apresentarem-se em formas de grânulos, esferas, cilindros, fibras e membranas (Brito et al., 2021).

A maioria dos métodos descritos na literatura para o desenvolvimento das nanopartículas usam solventes tóxicos que causam danos ambientais e aos sistemas biológicos (Jiang et al., 2020). Para diminuir os riscos ambientais e aos seres vivos, a síntese verde se apresenta como alternativa viável e eficiente no desenvolvimento das nanopartículas (Bruniera et al., 2020). Este processo de síntese utiliza enzimas, proteínas, polissacarídeos, aminoácidos e metabólitos presentes em biomassas vegetais como extratos de cascas, folhas, sementes e polpa (Rani et al., 2020).

O uso de métodos não tóxicos ecológicos e de baixo custo despertou o interesse de pesquisadores para síntese verde de nanopartículas metálicas utilizando extratos vegetais (Naseer et al., 2022). Pois as biomoléculas presentes nas plantas reduzem ativamente os íons metálicos, atuam como agentes protetores e estabilizadores das nanopartículas (Rai, 2013). A síntese verde além de ser um processo rápido e econômico não requer produtos tóxicos e ainda é compatível com produtos biomédicos de aplicação farmacêutica (Bruniera et al., 2020).

Os extratos naturais provenientes dos organismos vivos e utilizados na síntese de AgNPs, possuem aninas, fenólicos, proteínas, pigmentos, alcaloides e outras substâncias com poder redutor. Tais compostos conferem maior vantagem em relação aos métodos químicos devido a capacidade de adesão das moléculas na superfície das nanopartículas, que lhes dão características especificas, como a potencialização de atividades antimicrobianas (Camacho-Jiménez et al., 2020).

A síntese em si é inicialmente intuitiva devido aos efeitos visuais gerados imediatamente a formação das nanopartículas de prata. As AgNPs, têm propriedades ópticas únicas que as fazem interagir com específicos comprimentos de ondas de luz que possibilita rápida e facilmente sua caracterização por meio de espectroscopia por Ultravioleta visível (UVVis) (Jiang et al., 2020). A morfologia e o tamanho da nanopartículas formadas, pendendo da preparação, da natureza do solvente, concentração do agente redutor e da temperatura (Siddiqi et al., 2018).

A curcumina é um composto ou um ingrediente presente no rizoma da planta (Curcuma longa) é um polifenol amplamente usado como corante natural tem ação antioxidante, antimicrobiana, anticoagulante e anticâncer (Carneiro \& Macedo, 2020) por estas ações, é muito usada em diversas atividades farmacológicas (Sabir et al., 2021). Os grupos fenólicos, 
$\beta$-dicetana e metila presentes na curcumina, além de ter um efeito autorregulador (Judaki et al., 2017) podem tem a capacidade de reduzir e estabilizar nanopartículas da prata a partir do nitrato de prata.

A utilização de extratos de plantas para a síntese da nanopartícula, baseados nos conceitos de química verde, se dá pela presença de compostos ativos na massa que facilitam a redução e estabilização. Dentre as plantas ricas nestes compostos encontra-se o cajueiro (Anacardium occidentale L.), planta nativa do Brasil com característica dos campos e das regiões costeiras do Norte e Nordeste (Novaes \& Novaes, 2021). Além, dos lipídeos fenólicos destacam-se os flavonoides, apigenina, kanferol, quercetina e agastiflavona que possibilitam a estabilização das nanopartículas.

Esta pesquisa teve como objetivo sintetizar, caracterizar e comparar a formação de nanopartículas de prata em extratos de Curcuma longa e Anacardium occidentale.

\section{Metodologia}

A pesquisa adotou a metodologia laboratorial de natureza quantitativa, observou os fenômenos e analisou os elementos encontrados com a finalidade de estabelecer relações quantitativas entre eles (Pereira et al., 2018).

Para obtenção do extrato de curcumina foram utilizadas $200 \mathrm{~g}$ de pó do rizoma da Curcuma longa macerado em álcool (70\%) na proporção de 1:5 por 24 horas. O extrato hidroalcoólico foi filtrado quatro vezes usando um filtro de algodão, por fim foi centrado em baixa pressão obtendo-se o extrato seco.

Para obtenção do extrato de cajueiro (Anacardium occidentale) foram utilizadas $200 \mathrm{~g}$ de pó das folhas, macerado em álcool (70\%) na proporção de 1:5 por 24 horas. O extrato hidroalcoólico foi filtrado quatro vezes usando um filtro de algodão, por fim foi centrado em baixa pressão obtendo-se o extrato seco.

Para a síntese das nanopartículas de prata foi usado $125 \mathrm{~mL}$ de nitrato de prata $1,0 \mathrm{mmol} / \mathrm{L}$, aquecido em agitação magnética numa chapa aquecedora. Ao atingir a ebulição foi gotejado gota a gota $5 \mathrm{~mL}$ de citrato de sódio $1 \%$ na solução de nitrato de prata. O gotejamento ocorreu numa taxa de 1 gota por segundo, sob aquecimento e agitação magnética constante.

Ao longo do gotejamento a mistura mudou de cor, indicando a redução da prata com formação de nanopartículas, ao atingir a coloração amarelo esverdeado, interrompeu-se o aquecimento da chapa aquecedora, mantendo-se em agitação magmática.

Ao atingir a temperatura ambiente $25^{\circ} \mathrm{C}$ (graus célsius) adicionou-se à mistura, uma solução de extrato de curcumina. Depois de 15 minutos, a solução nitrato de prata/citrato/curcumina foi armazenada num fraco âmbar. Repetiu-se o mesmo procedimento, separadamente, utilizando o extrato de folhas de cajueiro, obtendo-se uma solução de nitrato de prata/citrato/cajueiro.

As respectivas soluções (nitrato de prata/citrato/curcumina) e (nitrato de prata/citrato/cajueiro), foram submetidas à espectroscopia de infravermelho (UV-Vis) para confirmação e caracterização das nanopartículas de prata (AgNPs).

\section{Resultados e Discussão}

As nanopartículas de prata (AgNPs) foram formadas a partir no nitrato de prata $\left(\mathrm{AgNO}_{3}\right)$ acrescidos de citrato, extrato de curcumina (Curcuma longa) e extrato de cajueiro (Anacardium occidentale) como agentes redutores e estabilizadores. A estabilização das nanopartículas se atribui a presença de substâncias ativas, como os flavonoides presentes nos extratos que impossibilita a interação e consequentemente a agregação entre as mesmas.

A caracterização das nanopartículas por UV-Vis foi possível, devido à possibilidade das nanopartículas apresentarem absorção em regiões distintas do espectro visível, denominadas bandas plasmons, Ressonância de Plasmon de Superfície (SPR). Pois as nanopartículas metálicas têm comportamento óptico observado em decorrência da alteração do tamanho e da 
forma das partículas. As dimensões na escala nanométricas e a estabilização por citrato são semelhantes aos diversos estudos encontrados publicados sobre a formação de nanopartículas utilizando prata e citrato.

O processo de formação e estabilização das nanopartículas, a partir do cotejamento do citrato no nitrato de prata, se deu em fases de transição observado pela mudança de cores, indicando o aumento das nanopartículas de prata (Figura 1).

Figura 1 - Aspecto visual da mudança de coloração das soluções de nanopartículas de prata ao longo do gotejamento de citrato.
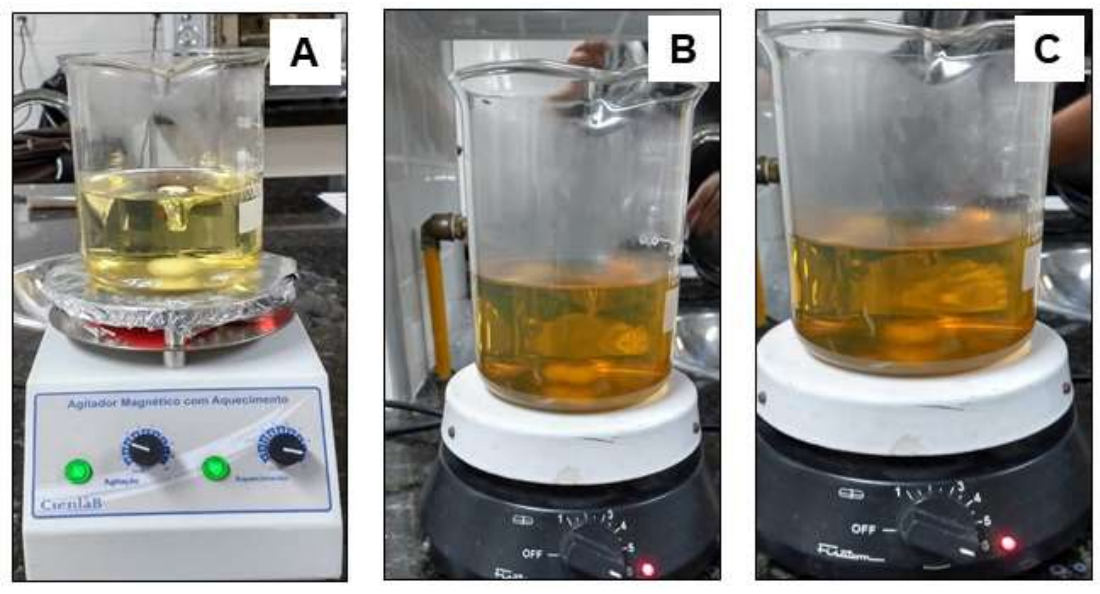

Fonte: Autores (2021).

A cor amarela observada nas AgNPs é resultante da absorção da radiação eletromagnética em ressonância com os plasmons de superfície. A cor amarela indica o tamanho e ausência de aglomeração das AgNPs, característica da solução padrão, que muda para uma coloração mais turva, e em seguida para uma coloração com tom de escura.

Os resultados encontrados convergem com diversos autores que afirmam ser possível produzir extratos com atividades redutoras e estabilizadoras, apenas com a utilização do processo hidroalcoólico dispensando, dessa forma, o uso de solventes mais tóxicos e consequentes nocivos ao meio ambiente e os seres vivos (Álvarez et al., 2017). O processo pelo método de síntese verde além de ser um processo rápido e econômico não requer produtos tóxicos e ainda é compatível com produtos biomédicos de aplicação farmacêutica (Bruniera et al., 2020).

A relação de UV-Vis para as duas análises, apontaram que a síntese de AgNPs em curcumin (Figura 2) e cajueiro (Figura 3), apresentaram um pico de absorção em 400nm - 450nm, atribuída a Ressonância Plasmônica de Superfície (SPR) ou oscilação ressonante dos elétrons AgNPs na presença de luz. Considerando que os dois extratos indicam ser satisfatórios para a síntese de AgNPS, pois a tamanho das nanopartículas formadas não variam drasticamente nas diferentes soluções.

O pico máximo e único de absorção na faixa SPR em 420nm (Figura 2), indica que houve formação de AgNPs com tamanho aproximado de $20 \mathrm{~nm}$ e formato esférico (Rani et al., 2020). O valor de absorbância indica o numero de nanopartículas presentes na solução, ou seja, quanto maior a absorção óptica maior será a quantidade de nanopartículas. 
Figura 2 - Espectro de absorção na região do Infravermelho referente às soluções de curcumina/citrato; nanopartícula de prata/extrato; nanopartículas de prata/citrato/curcumina.

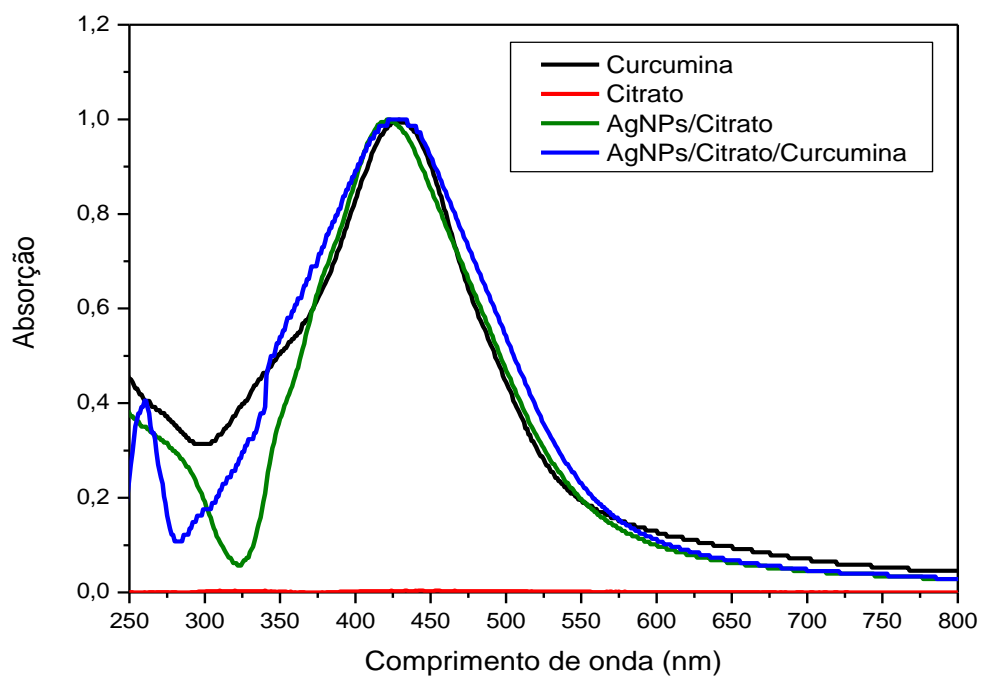

Fonte: Autores (2021).

No processo de síntese utilizando extrato de folhas de cajueiro (Anacardium occidentale), (Figura 3), O pico único de absorção na faixa SPR 420nm, apesar de tem um comprimento de onde mais longa indica também, que houve formação de AgNPs com tamanho aproximado de $20 \mathrm{~nm}$ e formato esférico (Rani et al., 2020). E que, o valor de absorbância indica o numero de nanopartículas presentes na solução, ou seja, quanto maior a absorção óptica maior será a quantidade de nanopartículas presentes na solução.

Figura 3 - Espectro de absorção na região do Infravermelho referente às soluções de caju; citrato; nanopartícula de prata/citrato; nanopartículas de prata/citrato/caju.

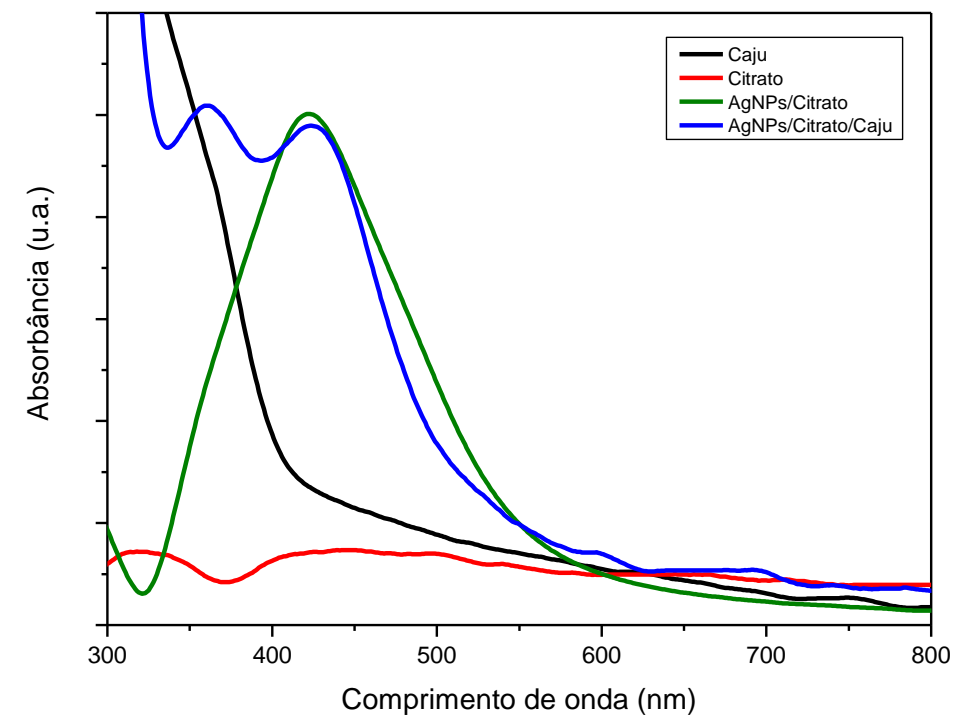

Fonte: Autores (2021).

De acordo com os resultados encontrados a partir da identificação da região ótima dos fatores para a síntese realizada neste estudo, pode-se observar que os dois extratos apresentaram condições ideais de acordo com a literatura, fato relacionado à ocorrência de pico na região em torno de 400-450nm. Resultados semelhantes foram encontrados no estudo de (Raja et al., 
2017). A diferença entre os resultados encontrados entre o experimento utilizando curcumin e o experimento utilizando extrato de cajueiro pode está na variação do tamanho da nanopartículas, visto que as bandas (SPR) são muito sensíveis ao tamanho das partículas, forma e a presença de biomoléculas no extrato biológico (Ruttkay-Nedecky et al., 2019).

\section{Discussão}

Nesta pesquisa foi possível discutir a síntese e caracterização das nanopartículas de prata como um metal que especificamente apresenta comportamentos magnéticos. Este material surge como um componente promissor amplamente utilizado em várias aplicações, por diversas áreas das ciências e tecnologia (Nelson (Durán et al., 2018). Tal interesse pode ser atribuído ao fato destes nanomateriais quando no seu estado natural, não apresenta propriedades químicas e físicas atribuídas às dimensões nanométricas.

Os metais são conhecidos por apresentarem propriedades ópticas específicas. Assim, as nanopartículas de prata sintetizadas nesta pesquisa e analisadas por meio do espectro de absorção utilizando espectrofômetro UV-Vis em uma faixa de comprimento de onda entre 250 e 800nm, apresentaram picos semelhantes aos trabalhos de (Dong et al., 2021) e (Houllou et al., 2019) com banda nítida, respectivamente, compatível com as excitações dos plasmons de superfície.

É importante ressaltar que os parâmetros para formação nanopartículas de prata, dependem da natureza dos materiais como concentração do nitrato de prata, concentração dos extratos vegetais, duração e temperatura do processo de síntese, entre outros. Estes fatores influenciam no tempo necessário para que ocorra a mudança da coloração das suspensões, bem como a intensidade dessas (Boriollo et al., 2020).

A síntese verde das nanopartículas de prata (Houllou et al., 2019), utilizando plantas e a possibilidade que essa abordagem de síntese proporciona, só é possível porque os extratos vegetais apresentam compostos fitoquímicos capazes de recobrir e estabilizá-las. No entanto, essa estabilidade no processo de síntese pode ser afetada por diferentes fatores, como pela espécie do solvente ou pela quantidade de agentes redutores.

Fatores abióticos relacionados com a sazonalidade também podem dificultar as padronizações das matérias primas e alterem físicas e quimicamente os componentes, implicando nos efeitos sobre as inconsistências da reprodutibilidade dos processos de síntese das nanopartículas (Huang et al., 2017).

\section{Conclusão}

Nesta pesquisa foram desenvolvidas e estabilizadas as nanopartículas de prata, com aspecto visual de cor amarelada, de forma esférica e dimensão em torno de $20 \mathrm{~nm}$ a partir da utilização de dois diferentes extratos de origem vegetal a curcumina e o extrato de folhas de caju (Anacardium occidentale). As nanopartículas sintetizadas em curcumina apresentou um pico de absorção no cumprimento de ondas entre 400nm - 450nm, atribuída a Ressonância Plasmônica de Superfície (SPR) ou oscilação ressonante dos elétrons AgNPs na presença de luz, semelhante o que foi observado nas nanopartículas sintetizadas no extrato de cajueiro.

Observou-se, ainda que para nanopartículas obtidas com extrato de curcumina, houve um pico bem definido e menor comprimento de onda, características da formação e estabilização de nanopartículas de prata pela síntese verde. Já o pico observado na síntese utilizando extrato de cajueiro, tem menor definição e onda mais alargada. Com estes resultados conclui-se que os extratos vegetais se mostraram eficientes no desenvolvimento e estabilização das nanopartículas por meio da síntese verde.

A pesquisa mostrou a necessidade de promover mais estudos utilizando o método verde para a síntese das nanopartículas. Considerado que se trata de uma tecnologia ecologicamente correta e sustentável, se faz necessário novos 
estudos no sentido de difundir o método e elevar a aplicação das nanopartículas de prata pelas diversas áreas das ciências como: biomedicina, biorremediação, cosmetologia, revestimento de produtos e utilização em embalagens.

\section{Referências}

Álvarez, A., Poejo, J., Matias, A. A., Duarte, C. M. M., Cocero, M. J., \& Mato, R. B. (2017). Microwave pretreatment to improve extraction efficiency and polyphenol extract richness from grape pomace. Effect on antioxidant bioactivity. Food and Bioproducts Processing, 106, 162-170. https://doi.org/10.1016/j.fbp.2017.09.007

Boriollo, M. F. G., Alves, V. E., Silva, T. A., Silva, J. J., Barros, G. B. S., Dias, C. T. S., Höfling, J. F., \& Oliveira, N. M. S. (2020). Decrease of the DXRinduced genotoxicity and nongenotoxic effects of Theobroma cacao revealed by micronucleus assay. Brazilian Journal of Biology. https://doi.org/10.1590/1519-6984.223687

Brito, J. E. de, Paranhos, C. N., Viana, D. dos S. F., Nunes, J. L., \& Cavalli, L. C. G. (2021). Genotoxicidade das nanoestruturas aplicadas em saúde: Uma revisão integrativa. Revista Eletrônica Acervo Saúde, 13(3), e6298. https://doi.org/10.25248/reas.e6298.2021

Bruniera, J. F. B., Gabriel-Silva, L., Goulart, R. S., Silva-Sousa, Y. T. C., Lara, M. G., Pitondo-Silva, A., \& Miranda, C. E. S. (2020). Green Synthesis, Characterization and Antimicrobial Evaluation of Silver Nanoparticles for an Intracanal Dressing. Brazilian Dental Journal, 31(5), 485-492. https://doi.org/10.1590/0103-6440202003897

Camacho-Jiménez, L., Álvarez-Sánchez, A. R., \& Mejía-Ruíz, C. H. (2020). Silver nanoparticles (AgNPs) as antimicrobials in marine shrimp farming: A review. Aquaculture Reports, 18, 100512. https://doi.org/10.1016/j.aqrep.2020.100512

Carneiro, J. A., \& Macedo, D. S. (2020). Cúrcuma: principios ativos e seus benefícios para a saúde.p., 14(87), 9.

Coura, M., Arroyos, G., Correia, L., \& Frem, R. (2018). Como obter nanopartículas de ouro usando suco de limão? Química Nova. https://doi.org/10.21577/0100-4042.20170202

Dong, B., Xue, N., Mu, G., Wang, M., Xiao, Z., Dai, L., Wang, Z., Huang, D., Qian, H., \& Chen, W. (2021). Synthesis of monodisperse spherical AgNPs by ultrasound-intensified Lee-Meisel method, and quick evaluation via machine learning. Ultrasonics Sonochemistry, 73 , 105485. https://doi.org/10.1016/j.ultsonch.2021.105485

Durán, N., Rolim, W., Durán, M., Fávaro, W., \& Seabra, A. (2018). Nanotoxicologia De Nanopartículas De Prata: Toxicidade Em Animais E Humanos. Química Nova. https://doi.org/10.21577/0100-4042.20170318

Echeverry-Chica, J. (2020). Nanopartículas de plata funcionalizadas in situ con D-limoneno: Efecto en la actividad antibacteriana. 14.

Houllou, L. M., De Souza, R. A. B., Malafaia, C. B., Paixão, D. L. M. da, Araújo, A. T. B. de, Silva, M. G., \& Duarte, G. C. S. (2019). Green synthesis of silver nanoparticles using leaf extract from Tabebuia roseoalba and T. pentaphylla. Journal of Environmental Analysis and Progress, 216-222. https://doi.org/10.24221/jeap.4.3.2019.2523.216-222

Huang, L., Wan, J., Wei, X., Liu, Y., Huang, J., Sun, X., Zhang, R., Gurav, D. D., Vedarethinam, V., Li, Y., Chen, R., \& Qian, K. (2017). Plasmonic silver nanoshells for drug and metabolite detection. Nature Communications, 8(1), 220. https://doi.org/10.1038/s41467-017-00220-4

Jiang, Q., Lin, Z., Gu, B., Pang, C., \& Wang, X. (2020). Green synthesis and immobilization of AgNPs by lumpy corn stalk as interlayer filling material for durable antibacterial. Industrial Crops and Products, 158, 112987. https://doi.org/10.1016/j.indcrop.2020.112987

Judaki, A., Rahmani, A., Feizi, J., Asadollahi, K., \& Hafezi Ahmadi, M. R. (2017). CURCUMIN IN COMBINATION WITH TRIPLE THERAPY REGIMES AMELIORATES OXIDATIVE STRESS AND HISTOPATHOLOGIC CHANGES IN CHRONIC GASTRITIS-ASSOCIATED HELICOBACTER PYLORI INFECTION. Arquivos de Gastroenterologia, 54(3), 177-182. https://doi.org/10.1590/s0004-2803.201700000-18

Naseer, Q. A., Xue, X., Wang, X., Dang, S., Din, S. U., Kalsoom, \& Jamil, J. (2022). Synthesis of silver nanoparticles using Lactobacillus bulgaricus and assessment of their antibacterial potential. Brazilian Journal of Biology, 82, e232434. https://doi.org/10.1590/1519-6984.232434

Novaes, T. E. R., \& Novaes, A. S. R. (2021). Análise dos potenciais medicinais do cajueiro (Anacardium occidentale Linn): Uma breve revisão. Research, Society and Development, 10(1), e41810111838. https://doi.org/10.33448/rsd-v10i1.11838

Pereira, A. S., Shitsuka, D. M., Parreira, F. J., \& Shitsuka, R. (2018). Metodologia da pesquisa científica. Metodologia da pesquisa científica, $1^{a}$ Edição, 119.

Rai, M. (2013). Nanobiotecnologia verde: Biossínteses de nanopartículas metálicas e suas aplicações como nanoantimicrobianos. Ciência e Cultura, 65(3), 44-48. https://doi.org/10.21800/S0009-67252013000300014

Raja, S., Ramesh, V., \& Thivaharan, V. (2017). Green biosynthesis of silver nanoparticles using Calliandra haematocephala leaf extract, their antibacterial activity and hydrogen peroxide sensing capability. Arabian Journal of Chemistry, 10(2), 253-261. https://doi.org/10.1016/j.arabjc.2015.06.023

Rani, P., Kumar, V., Singh, P. P., Matharu, A. S., Zhang, W., Kim, K.-H., Singh, J., \& Rawat, M. (2020). Highly stable AgNPs prepared via a novel green approach for catalytic and photocatalytic removal of biological and non-biological pollutants. Environment International, 143, 105924. https://doi.org/10.1016/j.envint.2020.105924

Ruttkay-Nedecky, B., Skalickova, S., Kepinska, M., Cihalova, K., Docekalova, M., Stankova, M., Uhlirova, D., Fernandez, C., Sochor, J., Milnerowicz, H., Beklova, M., \& Kizek, R. (2019). Development of New Silver Nanoparticles Suitable for Materials with Antimicrobial Properties. Journal of Nanoscience and Nanotechnology, 19(5), 2762-2769. https://doi.org/10.1166/jnn.2019.15867

Sabir, S. M., Zeb, A., Mahmood, M., Abbas, S. R., Ahmad, Z., \& Iqbal, N. (2021). Phytochemical analysis and biological activities of ethanolic extract of Curcuma longa rhizome. Brazilian Journal of Biology, 81(3), 737-740. https://doi.org/10.1590/1519-6984.230628 
Research, Society and Development, v. 10, n. 6, e11310615512, 2021

(CC BY 4.0) | ISSN 2525-3409 | DOI: http://dx.doi.org/10.33448/rsd-v10i6.15512

Siddiqi, K. S., Husen, A., \& Rao, R. A. K. (2018). A review on biosynthesis of silver nanoparticles and their biocidal properties. Journal of Nanobiotechnology, 16(1), 14. https://doi.org/10.1186/s12951-018-0334-5 